\title{
Expected value of the additional state in evaluating the method of quantification and uncertainty of additional states in an analytical model of grade I hypertension
}

\author{
Takeshi Uchikura ${ }^{1,2^{*}}$, Makoto Kobayashi ${ }^{3}$, Masayuki Hashiguchi ${ }^{1}$ and Mayumi Mochizuki ${ }^{1}$
}

\begin{abstract}
Background: In the construction of pharmacoeoconomic models, simplicity is desirable for transparency (people can see how the model is built), ease of analysis, validation (how well the model reproduces reality), and description. Few reports have described concrete methods for constructing simpler models. Therefore we focused on the value of additional states and uncertainty in disease models with multiple complications.

Objectives: The objective of this study was to examine the possibility of ranking additional states in disease models with multiple complications using a method for evaluating the quantification and uncertainty of additional states.

Methods: The expected value of additional states (EVAS) was formulated to calculate the value of additional states from the variation between analytic models using the net benefit method, and uncertainty was subtracted from the variation. We also verified the usefulness and availability of this method in grade I hypertension as a verification of the disease model. We assumed that stroke was recognized as an associated complication of hypertension in the basic model. In addition, stroke recurrence, coronary heart disease (CHD), and end-stage renal disease (ESRD) were assumed to represent other complications of hypertension. Ten thousand Monte Carlo simulations were performed, and the probability distribution was assumed to be the beta distribution in clinical parameters. The ranges of clinical parameters were $\pm 6.25 \%, 12.5 \%, 25 \%$, and $50 \%$ of the standard deviation from the mean value.
\end{abstract}

Results: The EVAS in complications of CHD showed the greatest uncertainty. In contrast, the EVAS of ESRD differed from stroke recurrence in the value ranking by uncertainty.

Conclusions: The EVAS has the potential to determine the ranking of additional states based on the quantitative value and uncertainty in disease models with multiple complications.

Keywords: Expected Value of the Additional State, Pharmacoeconomics, Hypertension, Quantification, Uncertainty

\section{Background}

Pharmacoeoconomic model analysis is conducted to combine various parameters (effect, side effects, quality of life [QOL], and cost data) using modeling techniques, e.g., the Markov model [1]. The construction of analytic models is a key issue affecting their results. The guidelines

\footnotetext{
* Correspondence: take-u@cmed.showa-u.ac.jp

'Division for Evaluation and Analysis of Drug Information, Faculty of Pharmacy, Keio University, 1-5-30 Shibakoen, Minato-ku, Tokyo 105-8512, Japan

${ }^{2}$ Department of Hospital Pharmaceutics, Showa University School of Pharmacy, 1-5-8 Hatanodai, Shinagawa-ku, Tokyo 142-8555, Japan Full list of author information is available at the end of the article
}

for modeling analysis refer to internal validity, e.g., the course of a disease or clinical process, and external validity, e.g., comparing model results with real-world results $[2,3]$. Thus, The Joint Report of the International Society for Pharmacoeconomics and Outcomes Research and the Society for Medical Decision Making (report of the ISPOR-SMDM Modeling Good Research Practices Task Force-2 [4]) mentions that the simplicity of models is desirable for ease of analysis, description, transparency (people can see how the model is built), and validation (how well the model reproduces reality) [5]. However, few 
reports have described concrete methods for constructing simpler models.

Generally, in the construction of analytical (disease) models, we assume that multiple complications may occur as the outcome of a specific disease. The complications in the analytical model are selected based on their importance (generally referred to as "a state"), but currently there is no evaluation method for that purpose. Furthermore, it is important to consider the influence of the uncertainty of the state itself, but that is not done at present. Therefore, if we could attempt to estimate the quantitative value of additional states in an analytical model and examine the possibility of ranking additional states in disease models with multiple complications, it would contribute to the establishment of useful analytical models. Therefore, in this study we attempted to estimate the quantitative value of additional states and rank those additional states, in addition to evaluating the robustness of ranking while considering uncertainty.

\section{Objectives}

The objective of this study was to examine the possibility of ranking additional states in disease models with multiple complications using a method for evaluating the quantification and uncertainty of additional states.

\section{Methods}

Method to evaluate quantification of the value of additional states and uncertainty of transition probabilities

We applied the net benefit method [6] to estimate the quantification of the value of additional states. This method assumes a net monetary benefit and a net health benefit in the same index using the threshold (e.g., 1 quality-adjusted life year [QALY] $=5$ million yen) of an incremental cost-effectiveness ratio, and evaluates intervention effects in the single index as the result of costeffectiveness analysis. This evaluation converts costs and QALY into the same index using a threshold ( $\lambda$ ) by applying the concept of net benefit and subsequently adds these values as components of the value of the states. Specifically, analytical model A is is the basic model, and analytical model B reflects the addition of a specific complication as an additional state to analytical model A. We estimated the value of the additional state as the change in the cost and effectiveness for the net benefit between the two models using Eq. (1):

Value of additional state $=\left(C_{B}-C_{A}\right) / \lambda+\left(E_{A}-E_{B}\right) \cdot \cdots \cdot$

where $C_{A}=$ the cost of analytical model $A, C_{B}=$ the cost of analytical model $B, \lambda=5,000,000$ yen (the value of 1 QALY), $E_{A}=$ the effectiveness (QALY) of analytical model $\mathrm{A}$, and $\mathrm{E}_{\mathrm{B}}=$ the effectiveness (QALY) of analytical model B.

Then, we defined the expected value of the additional state (EVAS) as the true value of the additional state, taking into account the robustness of the results with the uncertainty of the occurrence of the additional state, and estimated the value using Eq. (2). The uncertainty of the additional state based on the transition probabilities is calculated as the absolute value of the difference in the value of the additional state and a trial value in Monte Carlo simulations. Monte Carlo simulations are recognized as a useful method for performing multiple simulations of a model to obtain stable estimates of its variability $[1,7]$. In addition, the cumulative values were obtained from 10,000 Monte Carlo simulations of "the value of the additional state" and "the uncertainty of additional state."

$$
\begin{aligned}
\text { EVAS }= & \text { value of the additional state } \\
& - \text { uncertainty of that additional state } . . .
\end{aligned}
$$

Using Eq. (1), we can calculate each value of multiple complications (states) assumed in a specific disease based on costs and QALY and rank these complications (states) in the analytical model. Furthermore, we can estimate the robustness of the results based on the uncertainty of each state itself by estimating the EVAS from Eq. (2). Therefore, we can select a specific complication among multiple complications of a disease, because this method ranks multiple complications that consider the values of additional states and the robustness of the value of uncertainty of additional states.

\section{Disease model used for examining the ranking of the values of additional states}

We examined this evaluation method in hypertension, because it is a chronic model known to be associated with multiple complications. The basic model referred to the Markov model of grade I hypertension reported previously [8]. We did not assume an intervention effect for hypertension, because this model is less for pharmacoeconomic evaluation than for determining the value of additional states. The basic model covers stroke that is recognized as associated with hypertension [9]. In addition, stroke recurrence, coronary heart disease (CHD), and end-stage renal disease (ESRD) were assumed to represent other complications resulting from hypertension (Figure 1).

\section{Transition probabilities, QOL, and cost data on states in the disease model}

The hypothetical patients were assumed to be 55-year-old men, and the analytical time line was 10 years, because the Japan Public Health Center (JPHC) study [10] of 

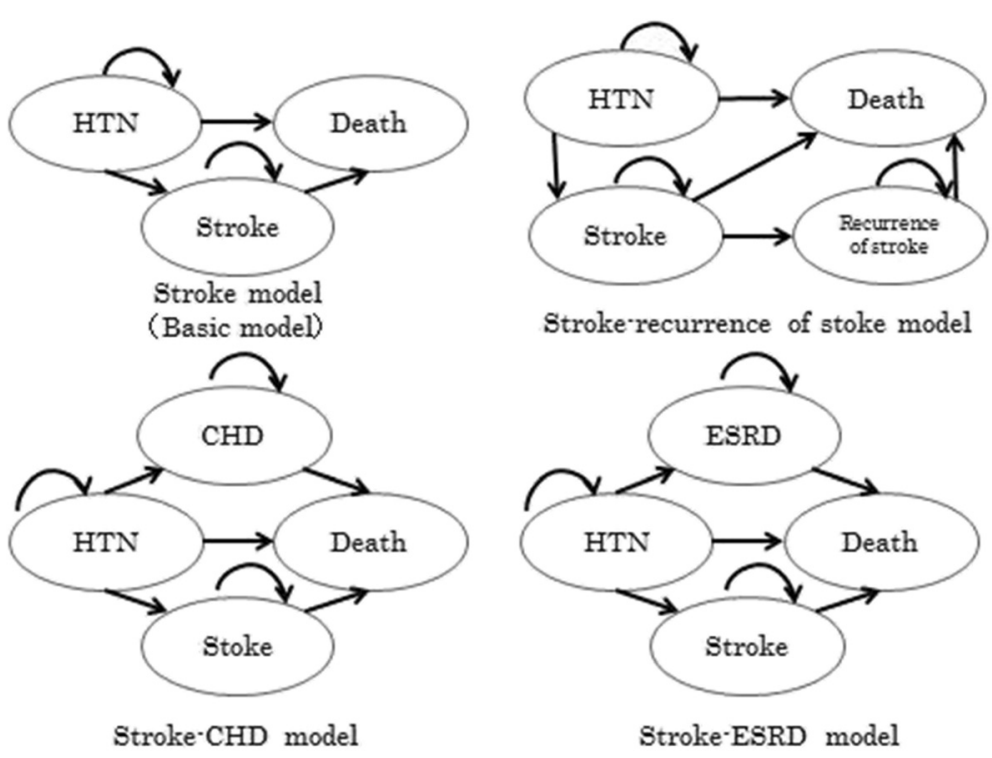

Strokerecurrence of stoke model

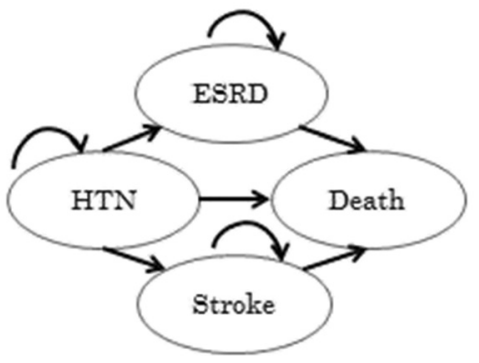

Stroke-ESRD model

Figure 1 The basic (stroke) model and each analytical model that assumed an additional state (complication). HTN: hypertension, CHD: coronary heart disease, ESRD: end-stage renal disease.

external validity enrolled men with grade I hypertension who had an average age of 56.0 years and the follow-up period was 11.0 years. We also used the annual incidences of stroke and $\mathrm{CHD}$ in patients with grade I hypertension (1000 persons/year) reported in the JPHC study [10]. The incidence of ESRD was also used as epidemiologic data in patients with grade I hypertension [11]. The rate of stroke recurrence and mortality after stroke, CHD, and ESRD were calculated from Japanese epidemiologic data [12-16]. The fifth-year parameter of stroke and the first-year parameter of mortality from CHD were used for the subsequent year and thereafter (Table 1). Nonstroke mortality was calculated based on the death rate of the Japanese population stratified by age [17].

The degree of disability after stroke was scored using the modified Rankin Scale (mRS) $[18,19]$, and allocations of patients were derived from the proportion of stroke type and mRS score at hospital discharge $[20,21]$. The degree of disability was assumed to remain constant over time.

Utilities for patients with disability after stroke and ESRD were based on Japanese data [22,23] and those after CHD were based on non-Japanese (UK) data [24] The utilities of health (hypertension alone) and death were assumed to be 1 and 0 , respectively (Table 2).

The cost of stroke treatment in the acute phase was obtained from the average number of hospitalized days based on the highest number of diagnostic procedure combinations in patients with each type of stroke [25]. The cost of recovery-phase rehabilitation facility care was calculated based on an average of 88 hospitalized days [26]. The annual cost of care was estimated from
Table 1 Clinical parameters

Annual incidence of stroke in grade I hypertension

(1000 people/year)

Men [10]

Annual incidence of $\mathrm{CHD}$ in grade I hypertension (1000 people/year)

Men [10]

Annual incidence of ESRD in grade I hypertension (1000 people/year)

Men [11]

Recurrence rate of stroke [12]*

First year

Second to fifth year

Mortality after stroke $[13]^{*} \dagger$

First year

$20.7 \%$

Second year

$6.7 \%$

Third year

$5.8 \%$

Fourth year

$5.9 \%$

Fifth year

$5.9 \%$

Mortality after CHD $[14,15]$

In hospital

$7 \%$

One year (1000 people/year)

Mortality after ESRD [16]

One year

$9.7 \%$

*Sixth year and thereafter use the same parameter as the fifth year.

${ }^{+}$Mortality after recurrence of stroke uses the same parameter. 
Table 2 Utility in patients after stroke, CHD, and ESRD [22-24]

\begin{tabular}{llllllll}
\hline & mRS 0* & mRS 1 & mRS 2 & mRS 3 & mRS 4 & mRS 5 & Death \\
\hline Stroke & $1^{*}$ & 0.83 & 0.67 & 0.45 & 0.24 & 0.09 & $0^{*}$ \\
CHD† & First year: & 0.68, second year or later: 0.72 & & \\
ESRD & 0.75 & & & & & &
\end{tabular}

*Assumed in this analysis.

†Data obtained from acute MI patients.

the amount of maximum payment for the categories of allowances for nursing care per month [27], which correspond to mRS scores of $2-5$, and it was calculated until death. In addition, we calculated work lost until the age of 65 years if the patients died at age 64 years or younger.

The cost of CHD treatment for myocardial infarction (MI) in the acute phase was obtained from actual hospitalization costs (an average of 25 hospitalized days) [28]. The costs of nursing care and work lost (excluding hospitalization in the acute phase) were not included, because CHD has relatively little affect on physical functions. The cost of ESRD treatment was assumed to be represented by that for dialysis [23] and conservative management [29] (Table 3; see Additional file 1).

The data on medical costs were obtained from official prices in Japan [25,30,31]. The costs of work loss reflected the average wages of each age cohort for regular employees [32].

\section{Discounting}

This study assumed that the discount rate was 3\% and $0 \%$ (no discounts) for reference values.

\section{External validity}

The external validity of analytical models compared the overall mortality from grade I hypertension patents in the JPHC Study [10] with the overall mortality of each analytical model.

\section{Statistical analyses}

Ten thousand Monte Carlo simulations were performed for each evaluation. The probability distribution for transition probabilities was assumed to be the beta distribution [33]. The ranges of transition probabilities were $\pm 6.25 \%$, $12.5 \%, 25 \%$, and $50 \%$ of the standard deviation (SD) from the mean. The simulation software TreeAge2013 (TreeAge Software, Inc. USA) was used.

\section{Results}

\section{Evaluation of external validity}

The overall mortality rate per year from the JPHC Study [10] was 0.0084 , in the basic model (stroke alone) it was 0.0086 , in the stroke-recurrence model it was 0.0087 , in the stroke-CHD model it was 0.0087 , and in the strokeESRD model it was 0.0086 .

\section{Evaluation of the value of additional states}

The values of additional states (complications) in the grade I hypertension model were 116 (92 for cost) for stroke recurrence, 295 (122 for cost) for CHD, 137 (100 for cost) for ESRD with dialysis, and 53 (16 for cost) for ESRD with conservative management (units are QALY). No differing trend was seen when no discount was assumed (Table 4).

\section{Evaluation of the EVAS}

The EVAS when uncertainty was considered tended to become smaller if the variable of the transition probabilities of the state grew larger. However, the ranking of additional states did not differ markedly when uncertainty was not taken into consideration. Therefore, the EVAS of CHD was the greatest of the additional states examined, even when the SD was $\pm 50 \%$, and the EVAS of ESRD with conservative management was the least in each case. In contrast, the EVAS of ESRD with dialysis was greater than that for stroke recurrence when uncertainty was not considered and reached 84 when the SD was $\pm 50 \%$. This estimate was less than the 89 for the EVAS of stroke recurrence when the SD was $\pm 25 \%$ (Table 4).

Table 3 Categorization of assumed costs related to medical treatment and others after stroke, CHD, and ESRD

\begin{tabular}{|c|c|c|c|c|c|c|c|}
\hline & & Severity & $\begin{array}{l}\text { Acute-phase } \\
\text { hospital care cost }\end{array}$ & $\begin{array}{l}\text { Recovery-phase } \\
\text { care cost }\end{array}$ & Ambulant treatment cost & $\begin{array}{l}\text { Nursing } \\
\text { care cost }\end{array}$ & Work lost* \\
\hline \multirow[t]{4}{*}{ After stroke } & First year & mRSO-1 & $\bullet$ & & $\bullet$ & & $\bullet$ \\
\hline & & mRS2-5 & $\bullet$ & $\bullet$ & $\bullet$ & $\bullet$ & $\bullet$ \\
\hline & Second year or later & mRSO-1 & & & $\bullet$ & & - (for clinic visits) \\
\hline & & mRS2-5 & & & $\bullet$ & $\bullet$ & $\bullet$ \\
\hline \multirow[t]{2}{*}{ After CHD } & First year & - & $\bullet$ & & $\bullet$ & & - (in hospital) \\
\hline & Second year or later & - & & & $\bullet$ & & - (for clinic visits) \\
\hline After ESRD & - & - & & & $\begin{array}{c}\text { - (hemodialysis or conservative } \\
\text { management) }\end{array}$ & & - (for clinic visit) \\
\hline
\end{tabular}


Table 4 Expected value of additional state (EVAS) of each complication

\begin{tabular}{|c|c|c|c|}
\hline & & Uncertainty** & EVAS $^{+}$ \\
\hline & & $( \pm 6.25 \% \mathrm{SD})$ & \\
\hline & $\begin{array}{c}\text { Value of } \\
\text { additional state* }\end{array}$ & $( \pm 12.5 \% \mathrm{SD})$ & \\
\hline & & $( \pm 25 \% \mathrm{SD})$ & \\
\hline & & $( \pm 50 \%$ SD) & \\
\hline & & $7(8)$ & 109 (132) \\
\hline Desurronge f ctrote & $116(140)$ & $13(16)$ & $102(124)$ \\
\hline Recurrence of stroke & $110(140)$ & $26(32)$ & 89 (108) \\
\hline & & $52(60)$ & $64(80)$ \\
\hline & & $15(18)$ & 280 (338) \\
\hline$C H \mathrm{O}$ & 205 & $30(36)$ & $265(320)$ \\
\hline CID & $295(350)$ & $60(72)$ & $235(284)$ \\
\hline & & $117(142)$ & $178(214)$ \\
\hline & & 7 (8) & 130 (159) \\
\hline ESPD - Dialycis troatment & $127(167)$ & $14(16)$ & $123(151)$ \\
\hline 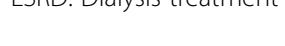 & (10) & $27(33)$ & 120 (134) \\
\hline & & $53(64)$ & $84(103)$ \\
\hline & & $3(4)$ & $50(61)$ \\
\hline Conconative troatmont & $53(65)+2-20$ & $6(7)$ & $47(58)$ \\
\hline conservative treatmemi & $53(03)$ & $12(15)$ & $41(50)$ \\
\hline & & $23(28)$ & $30(37)$ \\
\hline
\end{tabular}

Cumulative values of 10,000 Monte Carlo simulations.

CHD: coronary heart disease, ESRD: end-stage renal disease.

*The value of additional state $=\left(C_{B}-C_{A}\right) / \lambda+\left(E_{A}-E_{B}\right)$.

** Uncertainty $=$ an absolute value of the difference of the value of the

additional state (average) and the trial value of the Monte Carlo simulations.

${ }^{\dagger} \mathrm{EVAS}=$ the value of the additional state - the uncertainty (of the additional state).

Figures in parentheses indicate values when the discount rate is $0 \%$.

\section{Discussion}

In an attempt to construct a simpler model, this study ranked the importance of multiple complications in hypertension to estimate the value of additional states in a quantitative analytical model including transition probabilities, QOL, and cost data. In addition, we evaluated the robustness of the ranking of these values when considering the uncertainty of transition probabilities for the EVAS. To the best of our knowledge, this was the first experiment on quantification and ranking of the value of states along with uncertainty.

The ranking of the value of additional states was in the order CHD, ESRD with dialysis, stroke recurrence, and ESRD with conservative management in the analytical model of 55-year-old men with grade I hypertension. The differences in the assumed treatment in ESRD influenced the ranking of the value of the additional state, because the cost of dialysis treatment is 100 , but that of conservative treatment is 16 (the value of QALY conversion). Thus, the ranking of ESRD with dialysis and stroke recurrence will change with an assumption of the uncertainty of transition probabilities of the EVAS.

In the development of analytical models, the difference in internal validity and results of analytical models must be weighed $[34,35]$, particularly in analytical models constructed by academic groups in competitions like the Mount Hood Challenge Meeting [36]. Few reports referred to concrete methods for constructing simpler models. If our method enables the ranking of multiple complications in a specific model based on analytical values, more important and robust additional states could be identified. This would contribute to the development of a simpler model for pharmacoeconomic evaluation.

The limitations of this study were that: 1) only grade I hypertension was examined; 2 ) the setting of the threshold $(\lambda)$ in this method could be refined; and 3 ) the variation in all transition parameters was assumed to have the same range in a state. Although only grade I hypertension was considered, Eq. (2) shows that there would be no difference in the ranking of the value of the state based on the EVAS in other diseases. Therefore, this method has sufficient potential to be widely applied. The threshold $(\lambda)$ was set at 5 million yen for 1 QALY because 1 QALY in Japan was reported to be between approximately 5 and 6 million yen [37]. However, care must be taken because the results (the value of states) will change based on the setting of the threshold $(\lambda)$. Finally, the ranking of states (complications) may be different in some results from the EVAS based on variations in transition probabilities. The variation of all transition parameters was assumed to be in the same range in a state in this study. However, the actual uncertainty of transition probabilities can differ. It will be necessary to consider this in an actual analytical model in future.

\section{Conclusions}

This is the first report on devising a method for the evaluation and ranking of the quantitative value of additional states with uncertainty. It allows cumulative evaluation of transition probabilities, QOL, and cost data of a state using the EVAS. Generally, when we construct a pharmacoeconomic model, we consider states based on transition probabilities (e.g., incidence or mortality rate), but QOL and cost data are also components of a state. This evaluation method is useful because it quantifies the value of a state. In addition, the ranking of additional states in a disease model with multiple complications can be determined to identify which have less impact on a pharmacoeconomic model. The EVAS can therefore take into consideration the value of additional states with the uncertainty of transition probabilities. In the next phase of this continuing study, we plan to 
demonstrate the relation between the EVAS and pharmacoeconomic evaluation, i.e., the analytic results of interventions, and/or to examine the results in other chronic diseases that have multiple complications, e.g., chronic hepatitis $C$, etc., because we think that it is necessary to verify that this method will help to construct a simpler analytical model.

\section{Additional file}

Additional file 1: Table S1. Disease type in stroke [20]. Table S2. mRS scores for each type of stroke (partly modified) [21] and explanation of each mRS $[18,19]$. Table S3. Breakdown of acute hospital care costs $[25,28,29]$. Table S4. Breakdown of recovery-phase rehabilitation facility care costs $[26,29]$. Table S5. Breakdown of ambulant treatment costs after stroke and CHD $[30,31]$. Table S6. Breakdown of ambulant treatment costs after ESRD [23,29]. Table S7. Work lost (4 h) for clinic visit [32]. Table S8. Assumed nursing-care costs [27] for each mRS score.

\section{Competing interests}

The authors declare that they have no competing interest.

\section{Authors' contributions}

Conceived and designed the experiments: UT KM HM MM. Analyzed the data: UT. Wrote the paper: UT KM HM MM. All authors read and approved the final manuscript.

\section{Acknowledgments}

Yuma Higashino contributed to data collection.

\section{Author details}

${ }^{1}$ Division for Evaluation and Analysis of Drug Information, Faculty of Pharmacy, Keio University, 1-5-30 Shibakoen, Minato-ku, Tokyo 105-8512, Japan. ${ }^{2}$ Department of Hospital Pharmaceutics, Showa University School of Pharmacy, 1-5-8 Hatanodai, Shinagawa-ku, Tokyo 142-8555, Japan. ${ }^{3}$ CRECON Medical Assessment Inc, 2-12-15 Shibuya, Shibuya-ku, Tokyo 150-0002, Japan.

Received: 25 June 2014 Accepted: 30 October 2014

Published online: 28 January 2015

\section{References}

1. Stahl JE: Modelling methods for pharmacoeconomics and health technology assessment: An overview and guide. Pharmacoeconomics 2008, 26:131-148

2. National Institute for Health and Clinical Excellence: The Guidelines Manual 2012. http://www.nice.org.uk/article/PMG6/chapter/1\%20Introduction.

3. Fukuda T: Guidelines for analytical methods in health economics evaluation research. In Health and Labor Sciences Research Grant: Research on the Health Benefit Plan Applying Health Economics Evaluation. Tokyo; 2013.

4. Roberts M, Russell LB, Paltiel AD, Chambers M, McEwan P, Krahn M: Conceptualizing a model: A report of the ISPOR-SMDM Modeling Good Research Practices Task Force-2. Med Decis Making 2012, 32:678-689.

5. Eddy DM, Hollingworth W, Caro JJ, Tsevat J, McDonald KM, Wong JB, for the ISPOR-SMDM Modeling Good Research Practices Task Force: Model transparency and validation: a report of the ISPOR-SMDM Modeling Good Research Practices Task Force-7. Med Decis Making 2012, 32:733-743

6. Claxton K, Neumann PJ, Araki S, Weinstein MC: Bayesian value-of-information analysis. An application to a policy model of Alzheimer's disease. Int J Technol Assess Health Care 2001, 17:38-55.

7. Naversnik K, Rojnik K: Handling input correlations in pharmacoeconomic models. Value Health 2012, 15:540-549.

8. Uchikura T, Yokoi N, Hashiguchi M, Hashiguchi M, Mochizuki M: Pharmacoeconomic evaluation of hypothetical over-the-counter angiotensin-converting enzyme (ACE) inhibitors for the prevention of stroke in patients with grade I hypertension. Yakugaku Zasshi 2011, 131:571-580.

9. Japanese Society of Hypertension: Guidelines for the Management of Hypertension 2009. Tokyo: Japanese Society of Hypertension; 2009 [In Japanese].

10. Ikeda A, Iso H, Yamagishi $\mathrm{K}$, Inoue $\mathrm{M}$, Tsugane S: Blood pressure and the risk of stroke, cardiovascular disease, and all-cause mortality among Japanese: The JPHC study. Am J Hypertens 2009, 22:273-280.

11. Klag MJ, Whelton PK, Randall BL, Neaton JD, Brancati FL, Ford CE, Shulman NB, Stamler J: Blood pressure and end-stage renal disease in men. N Engl J Med 1996, 334:13-18.

12. Hata J, Tanizaki Y, Kiyohara Y, Kato I, Kubo M, Tanaka K, Okubo K, Nakamura H, Oishi Y, Ibayashi S, lida M: Ten year recurrence after first ever stroke in a Japanese community: The Hisayama study. J Neurol Neurosurg Psychiatry 2005, 76:368-372.

13. Investigative Commission on Measures of Stroke: Interim Report. Tokyo: Japanese Ministry of Health and Welfare, Health Service Bureau, Lifestyle-Related Disease Measure Office; 1999 [In Japanese].

14. Watanabe J, Iwabuchi K, Koseki Y, Fukuchi M, Shinozaki T, Miura M, Komaru T, Kagaya Y, Shirato K, Kitaoka S, Ishide N, Takishima T: Declining trend in the in-hospital case-fatality rate from acute myocardial infarction in Miyagi prefecture from 1980 to 1999. Jpn Circ J 2001, 65:941-946.

15. Japanese Coronary Artery Disease (JCAD) Study Investigators: Current status of the background of patients with coronary artery disease in Japan. Circ J 2006, 70:1256-1262.

16. Japanese Society for Dialysis Therapy, Committee of Renal Data Registry: An overview of regular dialysis treatment in Japan. http://docs.jsdt.or.jp/ overview/.

17. Japanese Ministry of Health, Labor and Welfare, Minister's Secretariat Statistics and Information Department: 2007 Abridged Life Table. Tokyo: Ministry of Health, Labor and Welfare; 2008. http://www.mhlw.go.jp/toukei/ saikin/hw/life/life07/index.html]. [In Japanese].

18. Van Swieten JC, Koudstaal PJ, Visser MC, Schouten HJ, van Gijn J: Interobserver agreement for the assessment of handicap in stroke patients. Stroke 1988, 19:604-607.

19. Shinohara Y, Minematsu K, Amano T, Ohashi Y: Reliablity of modified Rankin scale-introduction of a guidance scheme and a questionnaire written in Japanese. Jpn J Stroke 2007, 29:6-13 [In Japanese].

20. Kobayashi S (Ed): Japanese Stroke Data Bank 2009. Tokyo: Nakayama Shoten; 2009 [In Japanese]

21. Kobayashi S (Ed): Japanese Stroke Data Bank 2005. Tokyo: Nakayama Shoten; 2005 [In Japanese]

22. Noto S, Yanagi H, Tomura S: Measuring utilities for various functional outcomes after stroke, comparison of rating scale and time trade-off methods. Nippon Koshu Eisei Zasshi 2002, 49:1205-1216 [In Japanese].

23. Takura T, Kawanishi H, Minakuchi J, Nagake Y, Takahashi S: Cost-effectiveness analysis of on-line hemodiafiltration in Japan. Blood Purif 2013, 35:85-89.

24. Lacey EA, Walters SJ: Continuing inequality: gender and social class influences on self perceived health after a heart attack. J Epidemiol Community Health 2003, 57:622-627.

25. 2012 DPC electronic score list. [online]. [http://www.mhlw.go.jp/topics/2012/ 03/tp0305-02.html]. [In Japanese].

26. Koga M, Uehara T, Nagatsuka K, Yasui N, Hasegawa $Y$, Naritomi H, Okada $Y$, Minematsu K: Current role of convalescent rehabilitation units in community-based referral systems for stroke patients in Japan. Jpn J Stroke 2008, 30:735-743 [In Japanese]

27. Ministry of Health, Labor and Welfare: Public notice of no. 33 of 2000 (No. 258 revision). [In Japanese].

28. Fujimura $Y$ : Evaluation of the diagnosis procedure combination payment system: from the viewpoint of patients, medical and co-medical workers, and managements. Jpn J Political Economy Health Health Care 2010, 29:29-40 [In Japanese].

29. Ikeda S, Kobayashi M: Prediction of long-term prognosis for diabetes patients. Development of risk simulation software for cost-effectiveness analysis. Bunshi Touyoubyougaku no Shinpo; 2003:191-194 [In Japanese].

30. Medical fees scoreboards (Ikashinryohosyu Tensuhyo). 2012th edition. Tokyo: Shakai Hoken Kenkyujyo; 2012 [In Japanese].

31. National Health Insurance Price Dictionary (Hokenyaku Jiten). 2012th edition. Tokyo: Jiho, Inc; 2012 [In Japanese]. 
32. Ministry of Health, Labor and Welfare, Minister's Secretariat Statistics and Information Department: Basic Survey on Wage Structure, 2009. Tokyo: Ministry of Health, Labor and Welfare; 2008 [http://www.e-stat.go.jp/SG1/ estat/NewList.do?tid=000001011429]

33. Briggs AH, Weinstein MC, Fenwick EA, Karnon J, Sculpher MJ, Paltiel AD: ISPOR-SMDM Modeling Good Research Practices Task Force: Model parameter estimation and uncertainty: a report of the ISPOR-SMDM Modeling Good Research Practices Task Force-6. Med Decis Making 2012, 32:722-732.

34. Doan QV, Chiou CF, Dubois RW: Review of eight pharmacoeconomic studies of the value of biologic DMARDs (adalimumab, etanercept, and infliximab) in the management of rheumatoid arthritis. J Manag Care Pharm 2006, 12:555-569.

35. Ramos GF, Kuiper S, Dompeling E, van Asselt AD, de Grauw WJ, Knottnerus JA, van Schayck OC, Schermer TR, Severens JL: Structuring and validating a cost-effectiveness model of primary asthma prevention amongst children. BMC Med Res Methodol 2011, 11:150-163.

36. Palmer AJ, Clarke P, Gray A, Leal J, Lloyd A, Grant D, Palmer J, Foos V, Lamotte M, Hermann W, Barhak J, Willis M, Coleman R, Zhang P, McEwan P, Betz Brown J, Gerdtham U, Huang E, Briggs A, Carlsson KS, Valentine W, for the Mount Hood 5 Modeling Group: Computer modeling of diabetes and its complications: a report on the Fifth Mount Hood Challenge meeting Value Health 2013, 16:670-685.

37. Shiroiwa T, Sung YK, Fukuda T, Lang HC, Bae SC, Tsutani K: International survey on willingness-to-pay (WTP) for one additional QALY gained: what is the threshold of cost effectiveness? Health Econ 2010, 19:422-437.

\section{Submit your next manuscript to BioMed Central and take full advantage of:}

- Convenient online submission

- Thorough peer review

- No space constraints or color figure charges

- Immediate publication on acceptance

- Inclusion in PubMed, CAS, Scopus and Google Scholar

- Research which is freely available for redistribution 